\title{
THE MARTIN BOUNDARY FOR RANDOM WALK
}

\author{
BY \\ P. NEY(1),(2) AND F. SPITZER(3)
}

1. Introduction. We shall give a concrete realization of the Martin boundary for a very special class of Markov processes (random walks in the terminology of [8]). Of course, we obtain the same boundary as that constructed by Doob [2] and Hunt [6] for arbitrary transient Markov chains with discrete time and countable state space. Their boundary is obtained by completing the state space with respect to a metric which depends on the asymptotic behavior of the relativized Green's function of the Markov process in question. Here, too, the main theorem will concern the manner in which the relativized Green's function behaves as a point of the state space "tends to the boundary"- however, we shall be able to provide a geometric interpretation of the boundary and of the process of convergence to this boundary. For one-dimensional random walk ourconstruction was given by Doob, Snell, and Williamson [3]; there the boundary reduces to at most two points (the end points of a convex set, i.e., an interval determined by the transition function). The convex set replacing the interval in [3] when the dimension is $d>1$ has been carefully studied by Hennequin [5], and therefore, we face only one major difficulty - that of generalizing the so-called renewal theorem, which determines the asymptotic behavior of the Green's function, from dimension $d=1$ to $d>1$.

The boundary construction consists of two parts, the first of which is quite trivial, being independent of the random process. Let $R$ denote the space of lattice points $x=\left(x^{1}, x^{2}, \cdots, x^{d}\right)$ with integer-valued coordinates. The distance of $x$ from the origin is denoted by $|x|$. The mapping

$$
x \rightarrow \frac{x}{1+|x|}
$$

maps $R$ on a countable subset $S$ of the $d$-dimensional unit ball, and clearly $R$ and $S$ are homeomorphic (in the discrete topology). Now we may complete $S$ in the Euclidean metric to $S=S \cup \partial S$, where $\partial S$ is the unit sphere in $d$-space. If now $R$ is also completed, with respect to the metric

$$
\rho(x, y)=\left|\frac{x}{1+|x|}-\frac{y}{1+|y|}\right|,
$$

Received by the editors October 20, 1964.

(1) Supported by the National Institute of Health.

(2) Now at the University of Wisconsin, Madison, Wisconsin.

(3) Supported by the National Science Foudation at Cornell University. 
then the completion $\bar{R}=R \cup \partial R$ is clearly homeomorphic to $\bar{S}$, and $\partial R$ is homeomorphic to $\partial S$.

The second construction, which will consist in exhibiting still a third homeomorphic image of the boundaries $\partial R$ and $\partial S$, depends on the random walk in question. The random walk is the Markov chain with state space $R$, uniquely determined by a given transition function $P$ satisfying the conditions

$$
\begin{aligned}
& 0 \leqq P(x, y)=P(0, y-x), \quad x, y \in R, \\
& \sum_{x \in R} P(0, x)=1 .
\end{aligned}
$$

The iterates of $P$ are defined by

$$
\begin{aligned}
P_{0}(x, y) & =1 \text { if } x=y, \quad 0 \text { otherwise, } \\
P_{n+1}(x, y) & =\sum_{t \in R} P_{n}(x, t) P(t, y), \quad n \geqq 0 .
\end{aligned}
$$

We shall also assume that for each $x \in R$

$$
P_{n}(0, x)>0 \text { for some } n,
$$

which may depend on $x$; further, that the mean vector $\mu$ exists and does not vanish, i.e.,

$$
\sum_{x \in R}|x| P(0, x)<\infty, \quad \mu=\sum_{x \in R} x P(0, x) \neq 0 .
$$

Finally, we define a real-valued function $\phi$ on $d$-dimensional Euclidean space by

Let

$$
\phi(u)=\sum_{x \in R} P(0, x) e^{x \cdot u} \leqq \infty .
$$

$$
\begin{aligned}
D & =[u \mid \phi(u) \leqq 1], \\
\partial D & =[u \mid \phi(u)=1] .
\end{aligned}
$$

$D$ is clearly nonempty, because $\partial D$ contains the origin, but we shall assume more; namely, that

$$
\text { every point of } \partial D \text { has a neighborhood in which } \phi \text { is finite. }
$$

Hennequin [5] has proved the following under assumptions (1.1) through (1.4) (actually he assumes that $P(0, x)=0$ except at finitely many points of $R$, which implies (1.4), but (1.4) suffices for his proof):

Lemma 1.1. The set $D$ is compact and convex, the gradient

$$
\operatorname{grad} \phi(u)=\sum_{x \in R} x e^{u \cdot x} P(0, x)
$$

exists everywhere on $D$ and does not vanish on its boundary $\partial D$. Furthermore, the mapping 


$$
u \rightarrow \frac{\operatorname{grad} \phi(u)}{|\operatorname{grad} \phi(u)|}
$$

determines a homeomorphism between $\partial D$ and $\partial S$.

To describe the main theorem let

$$
G(x, y)=\sum_{n=0}^{\infty} P_{n}(x, y), \quad x, y \in R,
$$

which converges since our random walk is transient in view of condition (1.3). Note also that $G$ is strictly positive in view of (1.2), so that the relativized Green's function $G(x, y) / G(0, y)$ is well defined.

THEOREM 1.2. Suppose that (1.1) through (1.4) are satisfied. For an arbitrary $x \in R$, define $f: \bar{R} \rightarrow$ real numbers by

$$
\begin{aligned}
& f(y)=\frac{G(x, y)}{G(0, y)} \text { for } \quad y \in R, \\
& f(\eta)=e^{\alpha(\eta) \cdot x} \text { for } \quad \eta \in \partial R,
\end{aligned}
$$

where $\alpha$ is the homeomorphism $\alpha: \partial R \rightarrow \partial D$ constructed in two steps by the mappings $\partial R \leftrightarrow \partial S$ and $\partial S \leftrightarrow \partial D$ described above. Then the function $f$ is continuous on $\bar{R}$.

Since continuity of $f$ restricted to $\partial R$ is obvious, the theorem is equivalent to its more intuitive

Corollary 1.3. Let $p$ be a point in $\partial S$, and $u$ be the unique solution in $\partial D$ of

$$
p=\frac{\operatorname{grad} \phi(u)}{|\operatorname{grad} \phi(u)|} .
$$

Let $x_{n}$ denote any sequence in $R$ such that $\left|x_{n}\right| \rightarrow \infty$ and $x_{n} /\left|x_{n}\right| \rightarrow p$. Then for any $x$ in $R$

$$
\lim _{n \rightarrow \infty} \frac{G\left(x, x_{n}\right)}{G\left(0, x_{n}\right)}=e^{u \cdot x} .
$$

REMARKs. (a) It is well known [3] that the exponentials $\exp (u \cdot x), u \in \partial D$, are the extreme points of the cone of non-negative regular functions, i.e., solutions of the equation

$$
\sum_{y \in R} P(x, y) f(y)=f(x), \quad x \in R
$$

Furthermore, every non-negative $P$-regular function $f$ has a unique (Poisson) representation 


$$
f(x)=\int_{\partial D} e^{u \cdot x} d \mu(u),
$$

where $\mu$ is a positive Radon measure on $\partial D$. The potential theoretical proof of this and related facts, which is contained in the general theory of Hunt [6], can be rendered almost elementary by use of the above theorem. In particular, it follows from the law of large numbers applied to the random walk $\boldsymbol{x}_{\boldsymbol{n}}$ that

$$
\lim _{n \rightarrow \infty} \frac{x_{n}}{n}=\mu, \quad \lim _{n \rightarrow \infty} \frac{x_{n}}{\left|x_{n}\right|}=\frac{\mu}{|\mu|}
$$

with probability one. Since

$$
\mu=\operatorname{grad} \phi(0),
$$

we obtain from Corollary 1.3 , with $u=0$, that

$$
\lim _{n \rightarrow \infty} \frac{G\left(x, x_{n}\right)}{G\left(0, x_{n}\right)}=1
$$

with probability one.

(b) In the case when the random walk has mean $\mu=0$, i.e., when (1.3) does not hold, it is well known (see for instance Hennequin [5]) that the set $D$ reduces to a single point (the origin), and that all non-negative regular functions are constants. The natural compactification of $R$ is then the one-point compactification, and one would expect that

$$
\lim _{|x| \rightarrow \infty} \frac{G(x, y)}{G(0, y)}=1, \quad y \in R .
$$

The weakest conditions under which (1.5) holds are not known. However, an asymptotic estimate for $G(0, x)$ (similar to estimates we shall need to prove Theorem 1.2, but far easier) obtained in [8, P 26.1 and Problem 5, Chapter VI] shows that (1.5) holds under the conditions (1.1), (1.2),

$$
\begin{gathered}
\mu=\sum_{x \in R} x P(0, x)=0, \\
\sum_{x \in R}|x|^{2} P(0, x)<\infty,
\end{gathered}
$$

and finally

$$
\lim _{|x| \rightarrow \infty}|x|^{d-2} P_{n}(0, x)=0 \quad \text { for } n \geqq 1 \text {. }
$$

The last condition is superfluous when the dimension $d=3$ or 4 .

2. Proof of Theorem 1.2. For each unit vector $p \in \partial S$ let $u$ denote the unique solution in $\partial D$ of 


$$
p=\frac{\operatorname{grad} \phi(u)}{|\operatorname{grad} \phi(u)|} \text {. }
$$

We now introduce the transition function

$$
P^{u}(x, y)=P(x, y) e^{u \cdot(y-x)}, \quad x, y \in R,
$$

and observe that it again satisfies conditions (1.1) through (1.4) under which we wish to prove the theorem. The mean vector of $P^{u}$ is

$$
\mu^{u}=\sum_{x \in R} x P^{u}(0, x)=\operatorname{grad} \phi(u),
$$

so that it is a positive multiple of $p$. The Green's function of $P^{u}$ is

$$
G^{u}(x, y)=\sum_{n=0}^{\infty} P_{n}^{u}(x, y)=G(x, y) e^{u \cdot(y-x)}
$$

Since our estimates of the asymptotic behavior of these Green's functions will require delicate central limit theorems, we need to introduce the second moment quadratic forms: for vectors $\theta$ in $d$-dimensional Euclidean space we shall write

$$
Q^{u}[\theta]=\left(\theta \cdot Q^{u} \theta\right)=\sum_{x \in R}\left|\left(x-\mu^{u}\right) \cdot \theta\right|^{2} P(0, x) .
$$

These quadratic forms are positive definite by conditions (1.2) and (1.4) (see Lemma 2.4). Their inverses will be denoted $\Sigma^{u}$, and the corresponding determinants $\left|Q^{u}\right|$ and $\left|\Sigma^{u}\right|=\left|Q^{u}\right|^{-1}$. Finally, for vectors $\theta$ in $d$-dimensional Euclidean space, $\langle\theta\rangle$ will denote the nearest lattice point in $R$.

The proof, in outline, will proceed as follows. First we require a local central limit theorem.

THEOREM 2.1. Suppose $P(x, y)$ satisfies conditions (1.1) through (1.4) in $§ 1$, and that in addition $P_{n}(0,0)>0$ for all sufficiently large $n$. Let

$$
A_{n}(x, \gamma, u)=\left(\frac{\left|x-n \mu^{u}\right|}{\sqrt{ } n}\right)^{\gamma}\left[(2 \pi n)^{d / 2} P_{n}^{u}(0, x)-\left|Q^{u}\right|^{-1 / 2} \exp \left(-\frac{1}{2 n} \Sigma^{u}\left[x-n \mu^{u}\right]\right)\right] \text {. }
$$

Then

$$
\lim _{n \rightarrow \infty} A_{n}(x, \gamma, u)=0
$$

uniformly for $x \in R, 0 \leqq \gamma \leqq 2 d, u \in \partial D$.

This result will be shown to imply

THEOREM 2.2. Under conditions (1.1) through (1.4)

$$
\lim _{t \rightarrow \infty}(2 \pi t)^{(d-1) / 2} G^{u}\left(x,\left\langle t \mu^{u}\right\rangle\right)=\left[\left|Q^{u}\right|\left(\mu^{u} \cdot \Sigma^{u} \mu^{u}\right)\right]^{-1 / 2}
$$

for each $x \in R$, uniformly for $u \in \partial D$. 
The uniformity in Theorem 2.1 is required to obtain uniformity in Theorem 2.2, which in turn is needed to complete the proof of Theorem 1.2, or rather its corollary, as follows: suppose that $x_{n} \in R$, and

$$
\frac{x_{n}}{\left|x_{n}\right|}=p_{n} \rightarrow p \in \partial S
$$

and define $u_{n} \in \partial D$ by

$$
p_{n}=\frac{\operatorname{grad} \phi\left(u_{n}\right)}{\left|\operatorname{grad} \phi\left(u_{n}\right)\right|}
$$

Then

$$
G\left(x, x_{n}\right)=G^{u_{n}}\left(x, x_{n}\right) \exp \left[-u_{n} \cdot\left(x_{n}-x\right)\right]
$$

Also

$$
G^{u_{n}}\left(x, x_{n}\right)=G^{u_{n}}\left(x,\left\langle t_{n} \mu^{u_{n}}\right\rangle\right)
$$

where

$$
t_{n}=\frac{\left|x_{n}\right|}{\left|\operatorname{grad} \phi\left(u_{n}\right)\right|} .
$$

As $n \rightarrow \infty$, we have $t_{n} \rightarrow \infty, u_{n} \rightarrow u$, and by simple continuity arguments

$$
\mu^{u_{n}} \rightarrow \mu^{u}, \quad Q^{u_{n}} \rightarrow Q^{u}, \quad\left|\Sigma^{u_{n}}\right| \rightarrow\left|\Sigma^{u}\right|, \quad\left|\operatorname{grad} \phi\left(u_{n}\right)\right| \rightarrow|\operatorname{grad} \phi(u)| .
$$

Therefore

$$
\lim _{n \rightarrow \infty} \frac{G\left(x, x_{n}\right)}{G\left(0, x_{n}\right)}=\lim _{n \rightarrow \infty} \exp \left[u_{n} \cdot x\right]=e^{u \cdot x},
$$

which proves Corollary 1.3 and hence Theorem 1.2.

Proceeding to the proof of Theorem 2.1 we shall first consider only its special case when the vector $u \in \partial D$ is the zero vector. The modifications necessary to yield uniformity in $u \in \partial D$ will be supplied subsequently. Thus we shall prove

THEOREM 2.3. Let $P$ be the transition function of a d-dimensional random walk satisfying (1.1),(1.2) and the conditions that $P_{n}(0,0)>0$ for all sufficiently large $n$, as well as the moment condition

$$
m_{2 k}=\sum_{x \in R}|x|^{2 k} P(0, x)<\infty .
$$

Then the function $A_{n}(x, \gamma, u)$ defined in the statement of Theorem 2.1 has the property that

$$
\lim _{n \rightarrow \infty} A_{n}(x, \gamma, 0)=0
$$


uniformly for $x \in R$ and $0 \leqq \gamma \leqq 2 k$. (Note that $P^{u}=P, \mu^{u}=\mu, \Sigma^{u}=\Sigma, Q^{u}=Q$ when $u=0$.)

RemarK. For the case $\gamma=0$ and $d=1$ this is the classical local central limit theorem [4, p. 231]). For $\gamma=2$ and $\mu=0$ it appears in [8], and for $\gamma=2$ and $d=1$ it was proved by W. Smith [7]. Cox and Smith [1] used it to prove the one-dimensional renewal theorem in much the same way that we shall use Theorem 2.1 to obtain Theorem 2.2.

Proof. Since

$$
\min \left\{A_{n}(x, 0,0) ; A_{n}(x, 2 k, 0)\right\} \leqq A_{n}(x, \gamma, 0) \leqq \max \left\{A_{n}(x, 0,0) ; A_{n}(x, 2 k, 0)\right\},
$$

it suffices to prove the theorem for $\gamma=0$ and $\gamma=2 k$. In terms of the characteristic function

$$
\psi(\theta)=e^{i \mu \cdot \theta} \sum_{x \in R} P(0, x) e^{i x \cdot \theta}
$$

we obtain from the Fourier inversion formula

$$
(2 \pi n)^{d / 2} P_{n}(0, x)=(2 \pi)^{-d / 2} \int_{\sqrt{ } n C} \psi(w / \sqrt{ } n) \exp [-i w \cdot(x-n \mu) / \sqrt{ } n] d w,
$$

where $C$ is the cube $\left\{w\left|w=\left(w_{1}, \cdots, w_{d}\right),\right| w_{i} \mid \leqq \pi\right.$ for $\left.i=1, \cdots, d\right\}, \sqrt{ } n C$ its magnification by $\sqrt{ } n$, and $d w$ Lebesgue measure in Euclidean $d$ space $E$. We now take $A<\infty, 0<\alpha<\pi$ and write

$$
(2 \pi n)^{d / 2} P_{n}(0, x)=I_{0}(n)+I_{1}(n, A)+I_{2}(n, A)+I_{3}(n, A, \alpha)+I_{4}(n, \alpha),
$$

where

$$
\begin{aligned}
I_{0}(n) & =(2 \pi)^{-d / 2} \int_{E} \exp \left[-\frac{1}{2} Q[w]\right] \exp [-i w \cdot(x-n \mu) / \sqrt{ } n] d w \\
I_{1}(n, A) & =(2 \pi)^{-d / 2} \int_{|w| \leqq A}\left[\psi^{n}(w / \sqrt{ } n)-\exp \left[-\frac{1}{2} Q[w]\right]\right] \exp [-i w \cdot(x-n \mu) / \sqrt{ } n] d w \\
I_{2}(n, A) & =-(2 \pi)^{-d / 2} \int_{|w|>A} \exp \left[-\frac{1}{2} Q[w]\right] \exp [-i w \cdot(x-n \mu) / \sqrt{ } n] d w \\
I_{3}(n, A, \alpha) & =(2 \pi)^{-d / 2} \int_{A<|w| \leqq \alpha \sqrt{ }} \psi(w / \sqrt{ } n) \exp [-i w \cdot(x-n \mu) / \sqrt{ } n] d w
\end{aligned}
$$

and

$$
I_{4}(n, \alpha)=(2 \pi)^{-d / 2} \int_{\{\alpha \sqrt{ } n \leqq|w| ; w \in \sqrt{ } n c\}} \psi^{n}(w / \sqrt{ } n) \exp [-i w \cdot(x-n \mu) / \sqrt{ } n] d w .
$$

A direct calculation shows that

$$
I_{0}(n)=|Q|^{-1 / 2} \exp \left[-\frac{1}{2 n} \Sigma[x-n \mu]\right] \text {. }
$$


This is the main term in the theorem, and it remains to show that the terms $I_{1}, I_{2}$, $I_{3}, I_{4}$ go to zero uniformly in $x$ as $n \rightarrow \infty$.

First we note that for $|w| \leqq A$ we have $\psi^{n}(w / \sqrt{ } n) \rightarrow \exp \left[-\frac{1}{2} Q[w]\right]$ uniformly in $w$ by the classical central limit theorem, and hence

$$
\left|I_{1}(n, A)\right| \leqq(2 \pi)^{-d / 2} \int_{|w| \leqq A} \mid \psi^{n}(w / \sqrt{ } n)-\exp \left[-\frac{1}{2} Q[w]\right] d w \rightarrow 0 \quad \text { as } n \rightarrow \infty .
$$

Secondly

$$
\left|I_{2}(n, A)\right| \leqq(2 \pi)^{-d / 2} \int_{|w|>A} \exp \left[-\frac{1}{2} Q[w]\right] d w,
$$

which can be made arbitrarily small by taking $A$ sufficiently large. Next, using the fact that $(1-\psi(\theta)) / Q[\theta] \rightarrow \frac{1}{2}$ as $|\theta| \rightarrow 0$ (see P 7.7 of [8]), we see that $\alpha$ can be chosen sufficiently small so that

Hence

$$
\left|\psi^{n}(w / \sqrt{ } n)\right| \leqq \exp \left[-\frac{1}{4} Q[w]\right] \text { for }|w / \sqrt{ } n| \leqq \alpha
$$

$$
\left|I_{3}(n, A, \alpha)\right| \leqq(2 \pi)^{-d / 2} \int_{|w|>A} \exp \left[-\frac{1}{4} Q[w]\right] d w,
$$

which can again be made arbitrarily small by taking $A$ sufficiently large.

Finally we note that it follows from $P 7.8$ of [8] that there is a $\delta>0$ such that $|\psi(\theta)|<1-\delta$ when $\theta \in\{\theta: \theta \in C,|\theta| \geqq \alpha\}$. Hence

$$
\left|I_{4}(n, \alpha)\right| \leqq n^{d / 2}(2 \pi)^{-d / 2} \int_{\{\theta: \theta \in C,|\theta| \geqq \alpha\}}\left|\psi^{n}(\theta)\right| d \theta \leqq(2 \pi n)^{d / 2}(1-\delta)^{n} .
$$

This proves the theorem for $\gamma=0$.

We turn to the case $\gamma=2 k$. Many of the points in the argument will be very similar to the case $\gamma=0$. As before we have

$$
\begin{aligned}
\frac{|x-n \mu|^{2 k}}{n^{k}}(2 \pi n)^{d / 2} P_{n}(0, x)= & \left(\frac{n}{2 \pi}\right)^{d / 2} \frac{|x-n \mu|^{2 k}}{n^{k}} \\
& \cdot \int_{C}\left\{\psi^{n}(\theta)\right\} \exp [-i \theta \cdot(x-n \mu)] d \theta .
\end{aligned}
$$

Now if $f$ is any function on the torus $C$ (i.e., of period $2 \pi$ ) with continuous $2 k$ th derivatives, then by Green's second identity applied $k$ times

$$
\begin{aligned}
|x|^{2 k} \int_{C} f(\theta) e^{-i x \cdot \theta} d \theta & =(-1)^{k} \int_{C} f(\theta) \Delta_{\theta}^{k}\left[e^{-i x \cdot \theta}\right] d \theta \\
& =(-1)^{k} \int_{C} e^{-i x \cdot \theta} \Delta_{\theta}^{k} f(\theta) d \theta
\end{aligned}
$$


where $\Delta_{\theta}=\sum_{i=1}^{d}\left(\partial^{2} / \partial \theta_{i}^{2}\right)$ is the Laplace operator, and $\Delta_{\theta}^{k}$ is its $k$ th iterate. Applying this idea to (2.1), and setting $w=\theta \sqrt{ } n$, we get

$\frac{|x-n \mu|^{2 k}}{n^{k}}(2 \pi n)^{d / 2} P_{n}(0, x)=(-1)^{k}\left(\frac{n}{2 \pi}\right)^{d / 2} n^{-k} \int_{C} \exp [-i \theta \cdot(x-n \mu)] \Delta_{\theta}^{k}\left[\psi^{n}(\theta)\right] d \theta$

$$
=(-1)^{k}(2 \pi)^{-d / 2} \int_{\sqrt{ } n C} \exp [-i w \cdot(x-n \mu) / \sqrt{ } n] \Delta_{w}^{k}\left[\psi^{n}(w / \sqrt{ } n)\right] d w .
$$

At this point in the proof of the case $\gamma=0$ we used the fact that $\psi^{n}(w / \sqrt{ } n)$ $\rightarrow \exp \left[-\frac{1}{2} Q[w]\right]$ uniformly for $|w| \leqq A$. This time we need instead that

(2.3) $\lim _{n \rightarrow \infty} \Delta_{w}^{k}\left[\psi^{n}(w / \sqrt{ } n)\right]=\Delta_{w}^{k} \exp \left[-\frac{1}{2} Q[w]\right]$ uniformly for $|w| \leqq A$.

This can be shown by expanding the derivative on the left and then taking limits exactly as in formulas (4) and (5) of the proof of P 7.10 in [8], where the details appear for the case $\gamma=2$. The general case involves no new ideas. Note that the assumption $m_{2 k}<\infty$ is used at this point.

We now decompose (2.2) as follows. Write

$$
\frac{|x-n \mu|^{2 k}}{n^{k}}(2 \pi n)^{d / 2} P_{n}(0, x)=J_{0}(n)+J_{1}(n, A)+J_{2}(n, A)+J_{3}(n, A, \alpha)+J_{4}(n, \alpha),
$$

where

$$
\begin{aligned}
J_{0}(n)= & (-1)^{k}(2 \pi)^{-d / 2} \int_{E} \exp [-i w \cdot(x-n \mu) / \sqrt{ } n] \Delta_{w}^{k}\left[\exp \left[-\frac{1}{2} Q[w]\right]\right] d w, \\
J_{1}(n, A)= & (-1)^{k}(2 \pi)^{-d / 2} \int_{|w| \leqq A} \Delta_{w}^{k}\left[\psi^{n}(w / \sqrt{ } n)-\exp \left[-\frac{1}{2} Q[w]\right]\right] \\
& \cdot \exp [-i w \cdot(x-n \mu) / \sqrt{ } n] d w \\
J_{2}(n, A)= & (-1)^{k+1}(2 \pi)^{-d / 2} \int_{|w|>A} \Delta_{w}^{k}\left[\exp \left[-\frac{1}{2} Q[w]\right]\right] \\
& \cdot \exp [-i w \cdot(x-n \mu) / \sqrt{ } n] d w, \\
J_{3}(n, A, \alpha)= & (-1)^{k}(2 \pi)^{-d / 2} \int_{A<|w|<\alpha \sqrt{ } n} \Delta_{w}^{k}\left[\psi^{n}(w / \sqrt{ } n)\right] \\
& \cdot \exp [-i w \cdot(x-n \mu) / \sqrt{ } n] d w, \\
J_{4}(n, \alpha)= & (-1)^{k}(2 \pi)^{-d / 2} \int_{\{\alpha \sqrt{ } \leq|w| ; w \in \sqrt{ } n C\}} \Delta_{w}^{k}\left[\psi^{n}(w / \sqrt{ } n)\right] \\
& \cdot \exp [-i w \cdot(x-n \mu) / \sqrt{ } n] d w .
\end{aligned}
$$

Direct calculation shows that

$$
J_{0}(n)=n^{-k}|x-n \mu|^{2 k}|Q|^{-1 / 2} \exp \left[-\frac{1}{2 n} \Sigma[x-n \mu]\right],
$$


and it remains to show that the remainder terms go to zero uniformly in $x$. We proceed just as in the case $\gamma=0$. First replace each integrand in $J_{1}, J_{2}, J_{3}, J_{4}$ by its absolute value. This eliminates the dependence on $x$. Now (2.3) implies that $\lim J_{1}(n, A)=0$. Clearly $J_{2}(n, A)$ offers no new difficulties and can be made small by taking $A$ large.

Using $\mathbf{P} 7.7$ of [8] and the fact that $\psi(w / \sqrt{ } n)$ and its first $2 k$ derivatives are bounded in absolute value, we can show that there is an $\alpha>0$ such that for $|w / \sqrt{ } n| \leqq \alpha$

$$
\begin{aligned}
\left|\Delta_{w}^{k}\left[\psi^{n}(w / \sqrt{ } n)\right]\right| & \leqq \text { constant } \cdot\left|\psi^{n-2 k}(w / \sqrt{ } n)\right| \\
& \leqq \text { constant } \cdot \exp \left[-\frac{1}{4} Q[w]\right]
\end{aligned}
$$

Hence

$$
\left|J_{3}(n, A, \alpha)\right| \leqq(2 \pi)^{-d / 2} M \int_{|w|>A} \exp \left[-\frac{1}{4} Q[w]\right] d w
$$

for some $M<\infty$, which can again be made small by taking $A$ large.

Finally we use the first inequality of (2.4) and the previously referred to fact that $|\psi(\theta)|<1-\delta$ for $|\theta| \geqq \alpha, \theta \in C$ (where $\delta>0$ ) to yield

$$
\begin{aligned}
\left|J_{4}(n, \alpha)\right| & \leqq \text { constant } \cdot n^{d / 2}(2 \pi)^{-d / 2} \int_{\{|\theta| \geqq \alpha, \theta \in C\}}|\psi(\theta)|^{n-2 k} d \theta \\
& \leqq \text { constant } \cdot(2 \pi n)^{d / 2}(1-\delta)^{n-2 k} .
\end{aligned}
$$

This completes the proof of Theorem 2.3.

In order to deduce Theorem 2.1 from Theorem 2.3 it suffices to verify that the error terms $I_{1}$ through $I_{4}$ and $J_{1}$ through $J_{4}$, which were introduced in the proof of Theorem 2.3 , tend to zero uniformly in $u \in \partial D$ when $P$ is replaced by $P^{u}, Q$ by $Q^{u}, \psi(\theta)$ by $\psi^{u}(\theta)$, etc. This is easily verified with the aid of the following sequence of eight lemmas (2.4 through 2.11). They are all valid under the hypothesis that the random walk satisfies conditions (1.1) through (1.4) and in addition is strongly aperiodic, i.e., $P_{n}(0,0)>0$ for all sufficiently large $n$. Lemma 2.4 implies the uniform convergence to zero of $I_{1}$, Lemma 2.5 of $I_{2}$, and so forth, until Lemma 2.11 takes care of the last error term $J_{4}$.

LEMMA 2.4. The quadratic forms

$$
Q^{u}[\theta]=\sum_{x \in R}\left[\left(x-\mu^{u}\right) \cdot \theta\right]^{2} P(0, x)
$$

are uniformly positive definite, i.e., there exist positive constants $0<\lambda_{1} \leqq \lambda_{2}<\infty$ such that

$$
\lambda_{1}|\theta|^{2} \leqq Q^{u}[\theta] \leqq \lambda_{2}|\theta|^{2}, \theta \in E, u \in \partial D
$$


LeMma 2.5. Let

Then

$$
\psi^{u}(\theta)=\exp \left[-i \mu^{u} \cdot \theta\right] \sum_{x \in R} e^{i \theta \cdot x} P(0, x)
$$

$$
\lim _{n \rightarrow \infty}\left[\psi^{u}(w / \sqrt{ } n)\right]^{n}=\exp \left[-\frac{1}{2} Q^{u}[w]\right]
$$

uniformly for $w$ in any compact subset of $E$, and for $u \in \partial D$.

LEMMA 2.6. One can find an $\varepsilon>0$ and a constant $\lambda>0$ such that

$$
\left|\psi^{u}(w / \sqrt{ } n)\right|^{n} \leqq \exp \left[-\frac{1}{4} \lambda|w|^{2}\right], \quad u \in \partial D,
$$

when $|w / \sqrt{ } n|<\varepsilon$.

LeMmA 2.7. If $C$ is the cube defined in the proof of Theorem 2.3 and $N$ a neighborhood of 0 , then there exists some $\delta>0$ such that

$$
\left|\psi^{u}(\theta)\right|<1-\delta \text { for } \theta \in C-N \text {, }
$$

for all $u \in \partial D$.

LEMMA 2.8. There exist positive constants $\lambda, a$ and $b$ such that for every integer $k \geqq 0$

$$
\Delta_{w}^{k} \exp \left[-\frac{1}{2} Q^{u}[w]\right] \leqq a|w|^{b} \exp \left[-\frac{1}{2} \lambda|w|^{2}\right] .
$$

LEMMA 2.9. For each integer $k \geqq 0$

$$
\lim _{n \rightarrow \infty} \Delta_{w}^{k}\left[\psi^{u}(w / \sqrt{ } n)\right]^{n}=\Delta_{w}^{k}\left[\exp \left[-\frac{1}{2} Q[w]\right]\right]
$$

uniformly for $u \in \partial D$.

LEMMA 2.10. There exist positive constants $\varepsilon, \delta$, and c such that for $|w / \sqrt{ } n| \leqq \varepsilon$ and any integer $k \leqq n / 2$

$$
\left|\Delta_{w}^{k}\left[\psi^{u}(w / \sqrt{ } n)\right]^{n}\right| \leqq c \exp \left[-\delta|w|^{2}\right], \quad u \in \partial D
$$

LEMMA 2.11. Given any neighborhood $N$ of the origin in the cube $C$, there are positive constants $\delta$, and a such that

$$
\left|\Delta_{w}^{k}\left[\psi^{u}(w / \sqrt{ } n)\right]^{n}\right| \leqq a(1-\delta)^{n-2 k}
$$

when $w / \sqrt{ } n \in N, k \leqq n / 2, u \in \partial D$.

The proofs of these lemmas are omitted, being rather straightforward modifications of the preliminaries to the local central limit theorems in $\$ 7$ of [8]. 
The crucial fact, to be used repeatedly, is that condition (1.4) implies the existence of the moments of all orders of $P^{u}$, as well as the uniform boundedness, in $u \in \partial D$, of the moments of a fixed order. Lemma 2.4, which is crucial for all the subsequent lemmas with the exception of 2.7 , is of course dependent on the aperiodicity condition (1.2) which guarantees the positive definiteness of $Q^{u}$ for each fixed $u \in \partial D$. Only for Lemma 2.7 does one require, in addition, the strong aperiodicity condition that $P_{n}(0,0)>0$ for all sufficiently large $n$.

Proceeding now to the proof of Theorem 2.2 we shall first impose the additional assumption that the random walk is strongly aperiodic. Then, of course, not only $P_{n}(0,0)$ but also $P_{n}^{u}(0,0)$ is positive for sufficiently large $n$, independent of the point $u \in \partial D$. After completion of the proof this hypothesis may be removed by a simple continuity argument, given on p. 310 of [8] at the end of the similar but far easier renewal type theorem, P 26.1.

We shall decompose

$$
\begin{aligned}
& (2 \pi t)^{(d-1) / 2} \sum_{n=0}^{\infty} P_{n}^{u}\left(x,\left\langle t \mu^{u}\right\rangle\right) \\
& =\left\{\left|Q^{u}\right| \Sigma^{u}\left[\mu_{u}^{u}\right]\right\}^{-1 / 2}+H_{1}(t, u, k)+H_{2}(t, u, k)+H_{3}(t, u, k)
\end{aligned}
$$

where

$$
\begin{aligned}
& H_{1}(t, u, k)=(2 \pi t)^{(d-1) / 2} \sum_{n=\max (0, t-k \sqrt{ } t)}^{t+k \sqrt{ } t} P_{n}^{u}\left(x,\left\langle t \mu^{u}\right\rangle\right)-\left\{\left|Q^{u}\right| \Sigma^{u}\left[\mu^{u}\right]\right\}^{-1 / 2}, \\
& H_{2}(t, u, k)=(2 \pi t)^{(d-1) / 2} \sum_{n=1}^{t-k \sqrt{ } t-1} P_{n}^{u}\left(x,\left\langle t \mu^{u}\right\rangle\right), \\
& H_{3}(t, u, k)=(2 \pi t)^{(d-1) / 2} \sum_{n=t+k \sqrt{ } t+1}^{\infty} P_{n}^{u}\left(x,\left\langle t \mu^{u}\right\rangle\right) .
\end{aligned}
$$

Note that $P_{0}^{u}\left(x,\left\langle t \mu^{u}\right\rangle\right)=0$ when $t$ is sufficiently large, and that we simplify the notation by summing from $\alpha$ to $\beta$ when we mean the sum from the greatest integer in $\alpha$ to the greatest integer in $\beta$.

In terms of the function $A_{n}(x, \gamma, u)$ defined in the statement of Theorem 2.1,

$$
(2 \pi t)^{(d-1) / 2} P_{n}^{u}\left(x,\left\langle t \mu^{u}\right\rangle\right)=(2 \pi t)^{(d-1) / 2}(2 \pi n)^{-d / 2}\left|Q^{u}\right|^{-1 / 2}
$$

$$
\begin{gathered}
\cdot \exp \left\{-\frac{1}{2 n} \Sigma^{u}\left[\left\langle t \mu^{u}\right\rangle-x-n \mu^{u}\right]\right\} \\
+(2 \pi t)^{(d-1) / 2}(2 \pi n)^{-d / 2}\left|\frac{\sqrt{ } n}{\left\langle t \mu^{u}\right\rangle-x-n \mu^{u}}\right|^{\gamma} A_{n}\left(\left\langle t \mu^{u}\right\rangle-x, \gamma, u\right) .
\end{gathered}
$$

To simplify the notation introduce the vector

$$
z=z(x, t, u)=\left\langle t \mu^{u}\right\rangle-t \mu^{u}-x,
$$


and observe that $|z|$ is bounded uniformly in $t$ and $u$, for each fixed $x$. Next we decompose

$$
H_{1}(t, u, k)=H_{11}+H_{12}+H_{13}+H_{14}+H_{15},
$$

where

$$
\begin{aligned}
& H_{11}=\sum_{n=t-k t^{1 / 2}}^{t-t^{1 / 4}}(2 \pi t)^{(d-1) / 2}\left|Q^{u}\right|^{-1 / 2}(2 \pi n)^{-d / 2} \exp \left\{-\frac{1}{2 n} \Sigma^{u}\left[z+(t-n) \mu^{u}\right]\right\}, \\
& H_{12}=\sum_{n=t-t^{1 / 4}+1}^{t+t^{1 / 4}-1}\left[\text { same summand as in } H_{11}\right], \\
& H_{13}=\sum_{n=t+t^{1 / 4}}^{t+k t^{1 / 2}}\left[\text { same summand as in } H_{11}\right], \\
& H_{14}=(2 \pi)^{-1 / 2} t^{(d-1) / 2} \sum_{n=t-k t^{1 / 2}}^{t+k t^{1 / 2}} n^{-d / 2} A_{n}\left(\left\langle t \mu^{u}\right\rangle-x, \gamma, u\right), \\
& H_{15}=-\left\{\left|Q^{u}\right| \Sigma^{u}\left[\mu^{u}\right]\right\}^{-1 / 2} .
\end{aligned}
$$

Letting $v=n-t$, the error term $H_{11}$ takes on the form

$$
\begin{aligned}
H_{11}(t, u, k)= & (2 \pi)^{-1 / 2} t^{(d-1) / 2}\left|Q^{u}\right|^{-1 / 2} \sum_{v=-k t^{1 / 2}}^{-t^{1 / 4}}(t+v)^{-d / 2} \\
& \cdot \exp \left\{-\frac{1}{2}\left(\frac{v^{2}}{t+v}\right) \Sigma^{u}\left[\frac{z}{v}-\mu^{u}\right]\right\} .
\end{aligned}
$$

Setting

and

$$
\varepsilon_{t}(z, v, u)=\left(\frac{v^{2}}{t+v}\right) \Sigma^{u}\left[\frac{z}{v}-\mu^{u}\right]-\frac{v^{2}}{t} \Sigma^{u}\left[\mu^{u}\right]
$$

$$
r_{t}(z, v, u)=\left(\frac{t}{t+v}\right)^{d / 2} \exp \left\{-\frac{1}{2} \varepsilon_{t}(z, v, u)\right\},
$$

it is easily verified that we have

$$
\lim _{t \rightarrow \infty} r_{t}(z, v, u)=1
$$

uniformly for $u \in \partial D$ and for $v$ in $-k t^{1 / 2} \leqq v \leqq-t^{1 / 4}$.

We now rewrite (2.7) in the form

$$
H_{11}(t, u, k)=\left(2 \pi\left|Q^{u}\right|\right)^{-1 / 2} \sum_{\nu=-k t^{1 / 2}}^{-t^{1 / 4}} t^{-1 / 2} r_{t} \exp \left\{-\frac{v^{2}}{2 t} \Sigma^{u}\left[\mu^{u}\right]\right\},
$$

and claim that there exists an error function $R_{1}^{u}(k)$, such that 


$$
\lim _{t \rightarrow \infty} H_{11}(t, u, k)=\frac{1}{2}\left\{\left|Q^{u}\right| \Sigma^{u}\left[\mu^{u}\right]\right\}^{-1 / 2}+R_{1}^{u}(k),
$$

where

$$
\lim _{k \rightarrow \infty} R_{1}^{u}(k)=0 \quad \text { uniformly for } u \in \partial D .
$$

In view of (2.8) it suffices for the proof of (2.10) if we show that

$$
\begin{gathered}
\lim _{t \rightarrow \infty}\left(2 \pi\left|Q^{u}\right|\right)^{-1 / 2} \sum_{v=-k t^{1 / 2}}^{-t^{1 / 4}} t^{-1 / 2} \exp \left\{-\frac{v^{2}}{2 t} \Sigma^{u}\left[\mu^{u}\right]\right\} \\
=\frac{1}{2}\left\{\left|Q^{u}\right| \Sigma^{u}\left[\mu^{u}\right]\right\}^{-1 / 2}+R_{1}^{u}(k)
\end{gathered}
$$

uniformly in $u \in \partial D$, where $R_{1}^{u}(k)$ satisfies (2.11). For fixed $u$ we have

$$
\text { (2.13) } \lim _{t \rightarrow \infty} \sum_{v=-k t^{1 / 2}}^{-t^{1 / 4}} t^{-1 / 2} \exp \left\{-\frac{v^{2}}{2 t} \Sigma^{u}\left[\mu^{u}\right]\right\}=\int_{-k}^{0} \exp \left\{-\frac{1}{2} \rho^{2} \Sigma^{u}\left[\mu^{u}\right]\right\} d \rho \text {, }
$$

since the left side approximates the Riemann integral on the right. Set

$$
-R_{1}^{u}(k)=\int_{-\infty}^{-k} \exp \left\{-\frac{1}{2} \rho^{2} \Sigma^{u}\left[\mu^{u}\right]\right\} d \rho .
$$

One can conclude from Lemmas 1.1 and 2.4 that

$$
\Sigma^{u}\left[\mu^{u}\right] \geqq \lambda_{1}^{*}\left|\mu^{u}\right|^{2} \geqq \lambda_{2}>0,
$$

so that

$$
\left|R_{1}^{u}(k)\right| \leqq \int_{-\infty}^{-k} \exp \left\{-\frac{1}{2} \rho^{2} \lambda_{1}\left|\mu^{u}\right|^{2}\right\} d \rho \leqq \int_{-\infty}^{-k} \exp \left\{-\frac{1}{2} \rho^{2} \lambda_{2}\right\} d \rho,
$$

which implies that $R_{1}^{u}(k)$ satisfies condition (2.11).

The limit in (2.13) may now be written as

$$
(\pi / 2)^{1 / 2}\left\{\Sigma^{u}\left[\mu^{u}\right]\right\}^{-1 / 2}+R_{1}(k),
$$

and combining equations (2.12), (2.13), (2.16) we see that (2.12) is proved, provided we show that the convergence in (2.13) is uniform. For that purpose observe that

$$
\begin{aligned}
\sum_{v=-k t^{1 / 2}}^{-t^{1 / 4}} t^{-1 / 2} \exp \left\{-\frac{(v-1)^{2}}{2 t} \Sigma^{u}\left[\mu^{u}\right]\right\} & \leqq \int_{-k}^{0} \exp \left\{-\frac{1}{2} \rho^{2} \Sigma^{u}\left[\mu^{u}\right]\right\} d \rho \\
& \leqq \sum_{v=-k t^{1 / 2}}^{-t^{1 / 4}} t^{-1 / 2} \exp \left\{-\frac{(v+1)^{2}}{2 t} \Sigma^{u}\left[\mu^{u}\right]\right\},
\end{aligned}
$$

and therefore it will suffice to show that

$$
\lim _{t \rightarrow \infty} \sum_{v=-k t^{1 / 2}}^{-t^{1 / 4}} t^{-1 / 2}\left|\exp \left\{-\frac{(v+1)^{2}}{2 t} \Sigma^{u}\left[\mu^{u}\right]\right\}-\exp \left\{-\frac{(v-1)^{2}}{2 t} \Sigma^{u}\left[\mu^{u}\right]\right\}\right|=0
$$


uniformly for $u \in \partial D$. This is a simple consequence of the estimate (2.15), so that the estimate (2.10) for $H_{11}$ is now established.

In just the same way one proves that

$$
\lim _{t \rightarrow \infty} H_{13}(t, u, k)=\frac{1}{2}\left\{\left|Q^{u}\right| \Sigma^{u}\left[\mu^{u}\right]\right\}^{-1 / 2}+R_{1}^{u}(k) .
$$

Next we note that for some constant $C>0$

$$
\left|H_{12}(t, u)\right| \leqq C t^{-1 / 4} \rightarrow 0 \text { uniformly in } u \in \partial D \text { as } t \rightarrow \infty \text {. }
$$

Finally

$$
H_{14}(t, u, k) \leqq(2 \pi)^{-1 / 2} t^{(d-1) / 2}(t-k \sqrt{ } t)^{-d / 2} \cdot(2 k \sqrt{ } t) \cdot \max _{\{t-k \sqrt{ } t \leqq n \leqq t+k \sqrt{ } t\}} A_{n}\left(\left\langle t \mu^{u}\right\rangle-x, \gamma, u\right) .
$$

Hence it follows from Theorem 2.1 that

$$
\lim _{t \rightarrow \infty} H_{14}(t, u, k)=0 \text { uniformly for } u \in \partial D .
$$

The contributions from $H_{11}$ and $H_{13}$, which are independent of $k$, cancel the term $H_{15}$, so that combining (2.10), (2.17), (2.18), (2.19) we have

$$
\lim _{t \rightarrow \infty} H_{1}(t, u, k)=2 R_{1}^{u}(k) .
$$

Now we decompose $H_{2}(t, u, k)=H_{21}+H_{22}$, where

$$
\begin{aligned}
& H_{21}(t, u, k, \delta)=\sum_{n=1}^{\delta t}(2 \pi t)^{(d-1) / 2} P_{n}^{u}\left(x,\left\langle t \mu^{u}\right\rangle\right) \\
& \left.H_{22}(t, u, k, \delta)=\sum_{n=\delta t+1}^{t-k \sqrt{ } t-1} \text { [same summand as in } H_{21}\right]
\end{aligned}
$$

for some $0<\delta<1$.

Setting $\gamma=1+d / 2$ in equation (2.6) gives

$$
\begin{aligned}
& \begin{aligned}
H_{21}(t, u, k, \delta) \leqq & (2 \pi)^{-1 / 2}\left|Q^{u}\right|^{-1 / 2} \delta t \exp \left\{-\frac{1}{2 \delta t} \Sigma^{u}\left[\left\langle t \mu^{u}\right\rangle-x-[\delta t] \mu^{u}\right]\right\} \\
& +(2 \pi)^{-1 / 2} t^{(d-1) / 2} \sum_{n=1}^{\delta t} n^{(2-d) / 4}\left|\left\langle t \mu^{u}\right\rangle-x-n \mu^{u}\right|^{-(2+d) / 2}
\end{aligned} \\
& \cdot A_{n}\left(\left\langle t \mu^{u}\right\rangle-x, 1+\frac{d}{2}, u\right) .
\end{aligned}
$$

The first term on the right can be estimated by Lemma 2.4 in the form $\Sigma^{u}[\theta] \geqq \lambda|\theta|^{2}$. This yields an upper bound of the form $c_{1} t \exp \left\{-c_{2} t\right\}$ for some $c_{1}>0, c_{2}>0$. Furthermore, a simple calculation based on Theorem 2.1 shows that the second term is bounded above by a constant times $t^{-1 / 4}$. Hence 


$$
\lim _{t \rightarrow \infty} H_{21}(t, u, k, \delta)=0 \text { uniformly in } k \text { and } u \text {. }
$$

In treating $H_{22}$ we set $\gamma=2$. Letting $v=n-t$ and applying equation (2.6), one has

$$
\begin{gathered}
H_{22}(t, u, k, \delta)=(2 \pi)^{-1 / 2} t^{(d-1) / 2}\left|Q^{u}\right|^{-1 / 2} \sum_{v=-(1-\delta)^{*}}^{-k \sqrt{ } t}(t+v)^{-d / 2} \\
\cdot \exp \left\{-\frac{v^{2}}{2(t+v)^{2}} \Sigma^{u}\left[\frac{z}{v}-\mu^{u}\right]\right\} \\
+(2 \pi)^{-1 / 2} t^{(d-1) / 2} \sum_{n=\delta t+1}^{t-k \sqrt{ } t} n^{(1-d) / 2}\left|\left\langle t \mu^{u}\right\rangle-x-n \mu^{u}\right|^{-2} A_{t}\left(\left\langle t \mu^{u}\right\rangle-x, 2, u\right) .
\end{gathered}
$$

From (2.15) it follows that the first term on the right in (2.23) is bounded above by an expression of the form

$$
c_{1} \sum_{v=-(1-\delta) t}^{-k \sqrt{ } t} t^{-1 / 2} \exp \left\{-c_{2} \frac{v^{2}}{t}\right\}, \quad c_{1}>0, c_{2}>0,
$$

and the second term is bounded by

$$
c_{3} \sum_{n=\delta t+1}^{t-k \sqrt{ } t} \frac{\sqrt{ } t}{(t-n)^{2}}, \quad c_{3}>0 .
$$

The constants $c_{1}, c_{2}$ and $c_{3}$ are independent of $k$ and $\delta$, and the two sums (2.24) and (2.25) can be made arbitrarily small by choosing a large $k$. This may be seen by studying the approximating Riemann integrals. We may therefore summarize (2.22) through (2.25) by saying that

$$
\underset{t \rightarrow \infty}{\limsup }\left|H_{2}(t, u, k)\right| \leqq R_{2}(k),
$$

where $R_{2}(k)$ is independent of $u$ and tends to zero as $k \rightarrow \infty$. An analogous argument, applied to $\mathrm{H}_{3}$, shows that

$$
\lim _{t \rightarrow \infty} \sup \left|H_{3}(t, u, k)\right| \leqq R_{3}(k), \quad \lim _{k \rightarrow \infty} R_{3}(k)=0 .
$$

By applying (2.20), (2.26) and (2.27) to the decomposition (2.5), we see that Theorem 2.1 is proved in the strongly aperiodic case, and as mentioned at the outset, this suffices to give the result under conditions (1.1), (1.2), (1.3) and (1.4).

\section{BIBLIOGRAPHY}

1. D. R. Cox and W. L. Smith, $A$ direct proof of a fundamental theorem of renewal theory, Skand. Aktuarietidskr. 36 (1953), 139-150. 
2. J. L. Doob, Discrete potential theory and boundaries, J. Math. and Mech. 8 (1959), 433-458.

3. J. L. Doob, J. L. Snell and R. E. Williamson, Application of boundary theory to sums of

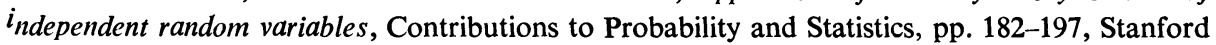
Univ. Press., Stanford, Calif., 1960

4. B. V. Gnedenko and A. N. Kolmogorov, Limit distributions for sums of independent random variables, Addison-Wesley, Cambridge, Mass., 1954.

5. P. L. Hennequin, Processus de Markoff en Cascade, Ann. Inst. H. Poincaré (2) 18 (1963), 109-196.

6. G. A. Hunt, Markoff chains and Martin boundaries, Illinois J. Math. 4 (1960), 313-340.

7. W. L. Smith, A frequency function form of the central limit theorem, Proc. Cambridge Philos. Soc. 49 (1953), 462-472.

8. F. L. Spitzer, Principles of random walk, Van Nostrand, Princeton, N. J., 1964.

\section{CORNELl UNIVERSITY,}

ITHACA, NEW YORK 